\title{
Conductance quantization as a new selective sensing mechanism in dendritic point contacts
}

\author{
Gennadii V. Kamarchuk ${ }^{1} \cdot$ Alexander P. Pospelov $^{2} \cdot$ Andriy V. Savytskyi $^{1} \cdot$ Anna O. Herus $^{1} \cdot$ Yurii S. Doronin $^{1}$. \\ Volodymyr L. Vakula ${ }^{1} \cdot$ Eric Faulques $^{3}$ (i)
}

(c) Springer Nature Switzerland AG 2019

\begin{abstract}
The diversity of techniques employed in modern sensing nanodevices is crucial for large-scale use of sensors in multifunctional technological cycles. We propose a new concept of selective detection of gases and liquids based on the formation of an original quantum system and registration of its energy states in dynamic mode using dendrite point contacts synthesized electrochemically in the probed medium. The in situ synthesis of nanosized dendrite point contacts is shaped by the cyclic switchover effect which takes place in an electrolyte in contact with the analyzed medium and results in consecutive cycles of the formation and destruction of an electrochemical gapless electrode system. Conductivity of such point contacts demonstrates quantum behavior driven by the shell effect which determines the geometry of their conducting channels. Temporal dependence of dendrite point contact electrical resistance measured in dynamic mode is characterized by a step-like structure which reflects the metastable quantum states of the system whose distribution can be presented in the form of a conductance histogram. The histogram is a unique fingerprint of the probed medium and can thus be used to unambiguously identify it. The dynamic mode scanning of the energy states of point contact quantum systems proposed here makes it possible to develop a universal method for selective detection of many gaseous and liquid media including such hard to detect substances as methane and rare gases. The new approach is expected to prove its efficiency in investigating quantum effects for various sensor applications and stimulate the development of the next generation of highly selective nanodevices.
\end{abstract}

Graphical abstract The new concept of selective detection of gases and liquids is based on the registration of quantum states of nanosized dendrite point contacts synthesized in the probed medium.

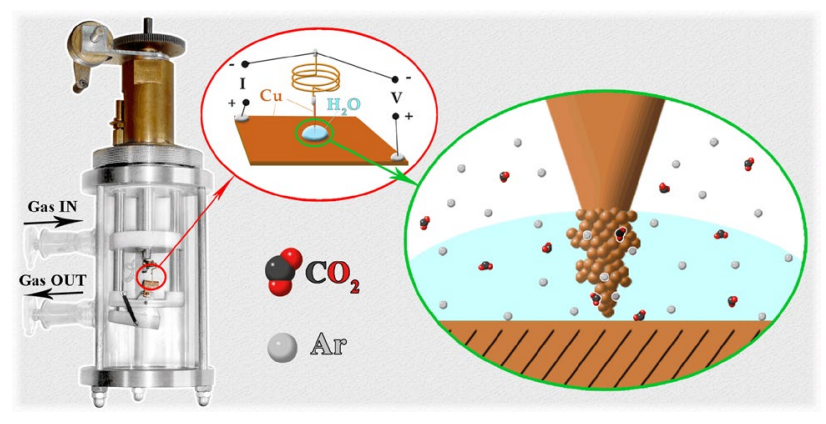

Eric Faulques, Eric.Faulques@cnrs-imn.fr|'Department of Spectroscopy of Molecular Systems and Nanostructured Materials, B. Verkin Institute for Low Temperature Physics and Engineering, National Academy of Sciences of Ukraine, 47 Nauky Ave., Kharkiv 61103 , Ukraine. 2Department of Physical Chemistry, National Technical University “Kharkiv Polytechnic Institute”, 2 Kyrpychov Str., Kharkiv 61002, Ukraine. ${ }^{3}$ Institut des Matériaux Jean Rouxel (IMN), CNRS, Université de Nantes, 2 rue de la Houssinière, BP 32229, 44322 Nantes Cedex 3 , France. 
Keywords Cyclic switchover effect - Dendrite point contact - Yanson point contact spectroscopy · Electronic transport in quantum wires $\cdot$ Gas detection

In recent years, the sensor technology has been one of the most dynamic areas of research. This has already resulted in a large variety of sensor devices, including nanosized ones, and applications. In addition to the traditional use of sensors to probe gaseous and liquid media [1-3], an ever-growing amount of attention is now being paid to new areas of sensor technology focused, e.g., on mobile phone, smart home, automotive and analytical chemistry applications [4-10]. Sensors have become an integral part of technologies aimed at improving the comfort level of human activities and medical diagnostics, monitoring the environment, providing better energy control and a higher level of industrial safety and security [11-15]. The diversity of operating principles implemented in modern sensing devices is an important prerequisite for large-scale use of sensors in multifunctional technological cycles. The operating principle of a sensor largely shapes its performance, functionality and ability to work reliably at a given minimal concentration of analyte. One of the promising trends in sensor research is the development of devices based on nanosized sensing elements which can bring new opportunities to considerably improve the sensor characteristics. The search for new innovative principles and their thorough testing can greatly stimulate the development of the next generation of sensing nanodevices. The purpose of this work is to propose an original concept of sensor detection based on the unique quantum properties of a dendrite point contact nanosystem, electrochemically synthesized in situ, under the action of a small amount of the probed substance.

Yanson point contacts (PCs) $[12,16]$ were chosen as operating elements of the proposed approach. They are well-known nanosized objects with amazing quantum properties and have been used as model systems to study conductance quantization [17-19]. Once immersed in a liquid conducting medium, the metallic channel of the contact turns into an electrochemical gapless electrode system (GES) (Fig. 1d) under applied voltage. GES was coined originally as electrochemical elongated element [20-22].

The experimental technique and methodology used in the study are described in detail elsewhere and are based on the simple Yanson's point contact technology which allows to prepare very swiftly copper PC electrodes [21, 22]. Dendrite point contacts (DPCs) were formed in the "needle-anvil" geometry shown in Fig. 1a. Initially, an electrochemically sharpened needle electrode was fixed on a spring damper above a flat electrode perpendicular to its surface. The DPCs were created in a special glass cell

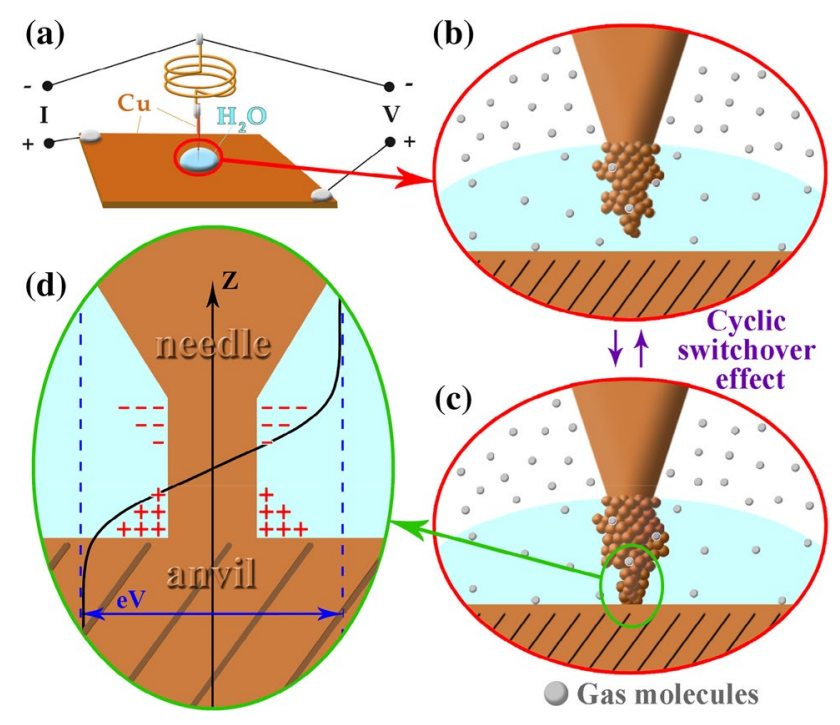

Fig. 1 Realization of the new concept of selective detection for nanosensor devices with dendrite point contacts operating as a sensing element. a Schematic presentation of the "needle-anvil" technique for Yanson dendrite-type PC preparation, b, c dendrite growth and dissolution in a drop of bi-distilled water (cyclic switchover effect) under gas action, the gas is represented by dots around and inside the water drop, $\mathbf{d}$ GES based on the conductive nanochannel of Yanson PC. $V$ voltage, I current, $Z$ axis of the contact

that contains an original device which permits a fine and smooth control of the interelectrode distance. Metallic dendrites were grown under electrochemical conditions at room temperature. Before the experiment, the glass cell was pumped out down to vacuum values of $10^{-2}$ Torr. Afterward, the gas to be investigated was injected into the cell. All measurements were made at atmospheric pressure. A four-probe arrangement was implemented to cancel the influence of current feeding leads. The electrical resistance of the sample (i.e., the needle-electrolyte-anvil system) was measured in alternating current according to Ref. [22]. To avoid possible influence of the background substances in the liquid medium on the obtained results, point contacts were placed in a drop of bi-distilled water (Fig. 1a), which was used as electrolyte. Despite the extremely low conductivity of bi-distilled water, electrochemical processes are observed to occur at the cathode and anode sections of the GES formed in the PC conductivity channel (see more details in Ref. [21]). As a result of the hydration process, some of the surface copper ions in contact with water migrate from lattice sites into the solution. This process weakens with time since the double 
electric layer formed at the phase boundary increasingly impedes the outflow of ions from the crystal. As a result, the studied environment that comes into contact with the surface of the point contact conductivity channel is not the deionized water, but the electrolyte solution containing the copper ions. Some equilibrium value of the concentration of these ions will determine the potential of the corresponding copper electrode. In the absence of voltage applied to the conductivity channel of the point contact, this potential has a constant value along the entire length of the channel. Once a certain current flows through the conductance channel, two areas occur at the phase boundaries: cathode, from the negative pole of the voltage source, and anode, from the opposite side. Therefore, for any nonzero biases, there will be a process of copper ion recovery and a formation of a crystal on the negatively polarized surface of the channel and an oxidation process with copper ions returning to the solution on the positively polarized surface until the complete interruption of direct conductance.

More specifically, GES is simply a specific type of electrochemical electrode system that makes it possible to electrochemically synthesize a large variety of nanostructures and functional materials. In contrast to the traditional electrochemical electrode system consisting of two or more electrodes separated by electrolyte and thus having no direct channel of electron conductivity, the GES is a monolithic conductor in an electric field with a potential uniformly distributed along its longitudinal axis. A macroscopic model of GES is merely a metallic conductor immersed into an electrolyte [23]. The main condition for the GES to function properly is the creation of a potential difference at its ends which is higher than the electrode system decomposition voltage [24] to trigger the electrochemical processes. This can easily be accomplished on the nanoscale level in a Yanson PC thanks to the unique potential distribution distinctive to this nanoobject [25, 26]. In the current flowing state of a system with two electrodes forming a Yanson PC (Fig. 1a), almost all potential drops over the conductivity channel of the point contact, and therefore, the electric field is concentrated in that area (Fig. 1d).

The cyclic switchover effect discovered by us in the process of DPCs synthesis [22] consists in cyclic phases of formation and dissolution of point contacts and can be an efficient tool to register the variety of quantum states of the system in dynamic mode (Fig. 1b, c). This is a cooperative effect arising both from the quantum conductance of a Yanson PC and from the unique electrochemical nature of its conductivity channel. Combining the fundamental characteristics of the nanoscale conductivity channel of Yanson PC [16] with oppositely directed electrochemical processes on its surface offers a means for selective detection of various analytes through quantum states of the PC system. The quantum shell effect is responsible for the synthesis of DPCs and for the metastable shapes of the PC conducting channel which in turn determine discreet values of conductance reflecting the energy states of the system. The cyclic switchover effect allows one to register these states in dynamic mode.

Making use of the opportunities provided by the cyclic switchover effect and the shell effect, we propose a new concept of selective detection for nanosensor devices based on the quantum properties of the sensing element (Fig. 1). The idea was inspired by the nonmonotonic dependence of conductance $G$ of a DPC on electrolyte concentration [22]. It follows from the dependence in Fig. 5 in Ref. [22] that even a slight variation in electrolyte concentration leads to a significant change in conductance and thus conductance histograms registered during the synthesis of DPCs. In accordance with the discovered dependence, any change in electrolyte concentration can affect the conditions under which the DPC is being synthesized and so determines the most probable set of its quantized energy states, which is thus supposed to depend on the environment in which the synthesis is performed. This means that gas molecules penetrating into the drop of electrolyte can lead to peculiar distributions of metastable DPC structures and energy states which are associated with them. In this case, the conductance histogram, which in fact sums up the probabilities of these quantum states, is a "fingerprint" of the point contact interaction process with the gas analyte. This means that any gas can be characterized by a unique conductance histogram for a DPC grown in its environment.

We measured and analyzed time dependences of the electrical resistance $R(t)$ of the electrode system and DPCs influenced by different gas media. For each of the gaseous medium studied, we obtained at least 10 dependences which yield reproducible conductance histograms. The histograms were derived to characterize the distribution of the point contacts' conductance using their maxima values. The histogram maximum corresponds to the most probable point contact under the conditions of the experiment. The confidence interval, according to the statistical calculations, did not exceed $3.5 \Omega$ with a $95 \%$ probability.

When current flows through the needle-electrolyte-anvil system (the needle is the cathode, the anvil is the anode, Fig. 1a), dendrites start growing in the electrode active areas of the needle surface with the highest density of electric field lines (Fig. 1b). If current continues to flow, a cyclic process [22] develops in the system after a period of some hundred milliseconds to several minutes (Fig. 1b, c): The PC resistance varies with time going through the phases of growth, reduction and stabilization (Fig. 2a, b). The phases are repeated many times, accounting for the 


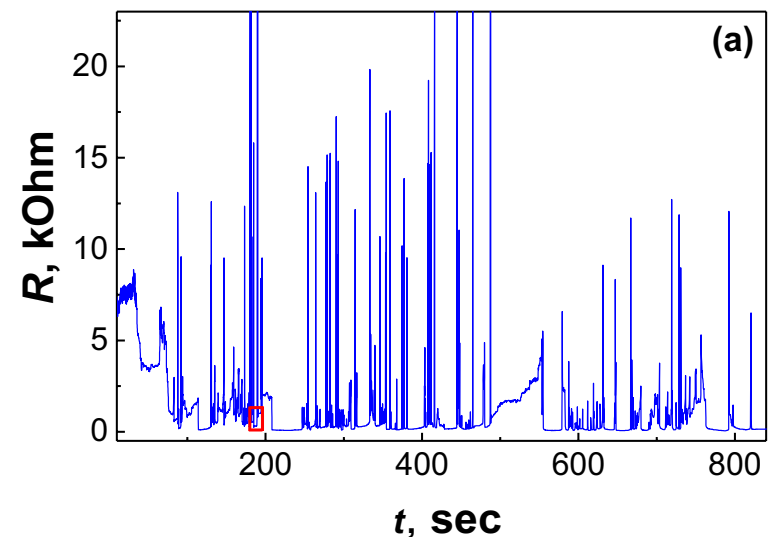

Fig. 2 Point contact resistance $R$ versus time $t$ in an $\mathrm{Ar}(80 \%)+\mathrm{CO}_{2}$ $(20 \%)$ medium. a General view of the dependence $R(t), \mathbf{b})$ a largescale image of $R(t)$ in the area within the rectangular section shown in Fig. 2a. Dashed lines mark conductance steps and metastable

cyclic changes in the physical and chemical properties of the object. The recurrence of the cyclic changes is automatic and requires no external influence. The variations in the interelectrode voltage during the self-oscillations of resistance show up as clearly visible steps in the $R(t)$ curves (Fig. 2b) and are attributed to electron conductance quantization and electronic shell effect in the DPCs [22].

Conductance temporal dependencies $G(t)$ of DPCs were obtained by inverse transformation of the experimental curves $R(t)$. The conductance steps emerging in a large number of successive cycles of DPC transformations were

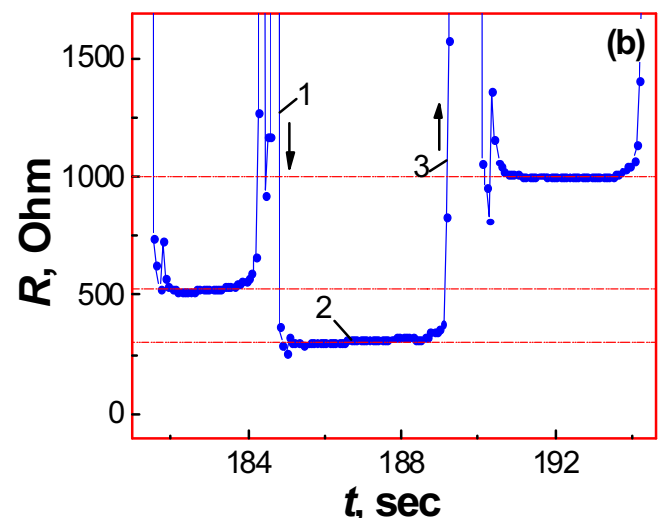

states of the contact; 1-phase of resistance reduction, 2-phase of resistance stabilization, 3-phase of resistance growth. Arrows indicate direction of the process. Current $I=20 \mu \mathrm{A}$

used to derive conductance histograms which display the distribution of the discrete PC conductance values. The procedure for processing the experimental data is described in detail in Ref. [22].

Conductance histograms from DPCs formed in the gaseous media of argon (100\%) and argon (80\%) $+\mathrm{CO}_{2}(20 \%)$ are shown in Fig. 3a, b. The histograms reflect the probability of certain quantum states in the conductance of DPCs formed under specific experimental conditions. As it follows from the Yanson point contact spectroscopy theory $[16,25]$, in spectral modes of current flow the resistance of
Fig. 3 Conductance histograms of dendrite copper PCs grown in the nanolayer of a drop of bi-distilled water near the copper surface under the action of a gas. a DPCs in a $100 \%$ Ar medium, b DPCs in an $\mathrm{Ar}(80 \%)+\mathrm{CO}_{2}(20 \%)$ medium, c DPCs in an $\mathrm{Ar}(99.99 \%)+\mathrm{CO}_{2}$ (0.01\%) medium, $\mathbf{d}$ DPCs in an $\operatorname{Ar}(99.99 \%)+\mathrm{CH}_{4}(0.01 \%)$ medium. $G$ conductance, $G_{0}$ conductance quantum, $n$ number of counts
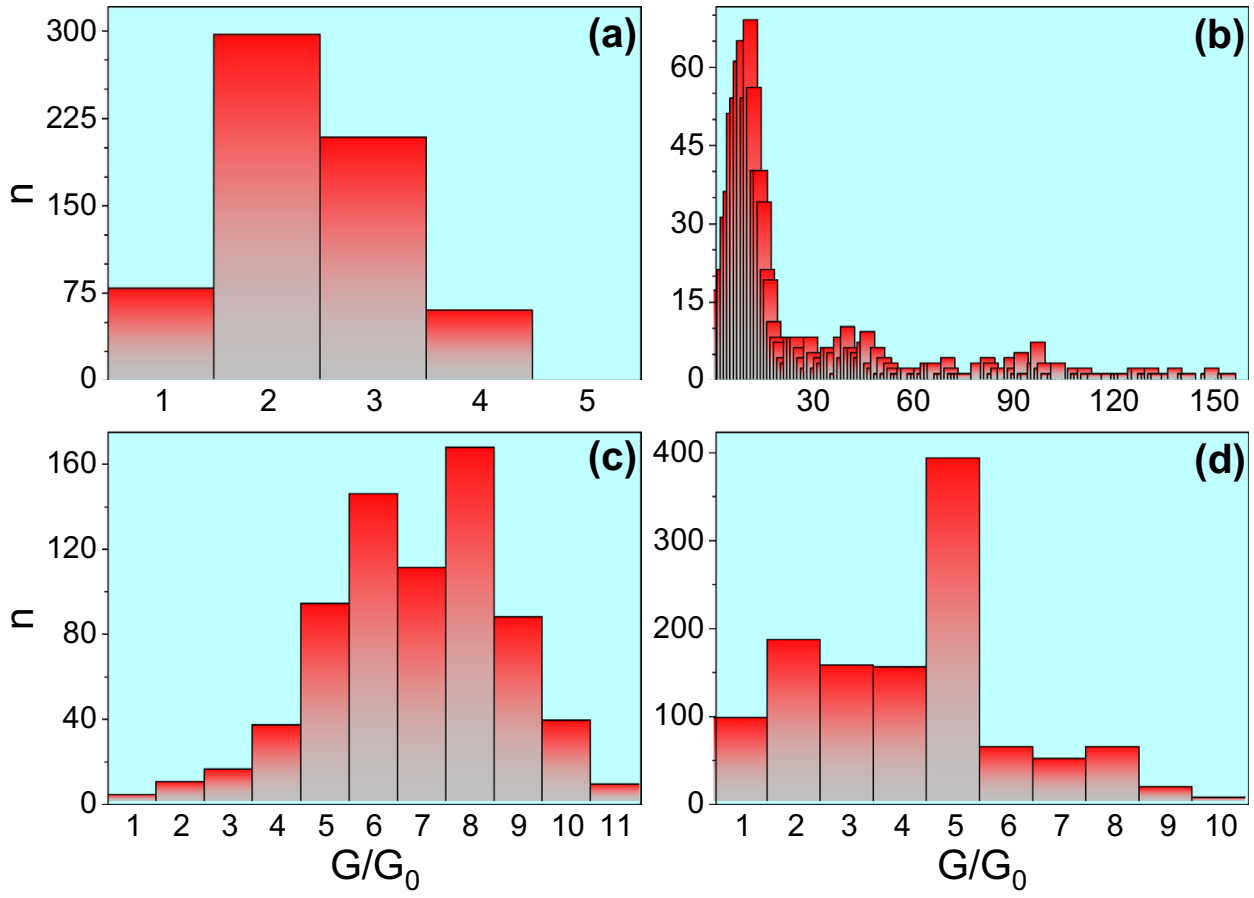
a PC is inversely proportional to its diameter. For a Yanson PC considered within the long-channel model, this means that any increase or decrease in its resistance in a range of $0-12.9 \mathrm{k} \Omega$, which corresponds to direct conductivity in the DPC, is accompanied by a respective drop or rise in the diameter of its conductivity channel. Taking into account that the conductance of a one-atom-sized contact with the resistance of $12.9 \mathrm{k} \Omega$ is approximately one conductance quantum $G_{0}$, we can conclude that the initial section of the conductance histogram corresponds to small-diameter contacts with dimensions close to those of one atom, while the section with large $G$ values contains information about large-diameter contacts. For a more detailed explanation, see Ref. [22]. Positions of the highest bars in the histograms, which correspond to the most probable metastable energy states, are related to specific cross sections of DPCs grown in the self-oscillatory process. The histogram maximum corresponds to the most probable point contact under the experimental conditions.

As one could expect, in the case of the pure rare gas the conductance histogram is quite simple, which implies, in accordance with Ref. [22], that argon only slightly influences the process of synthesis of DPCs. This influence is even less pronounced than that on the processes occurring during the synthesis of DPCs in a very weak electrolyte (see, for example, curve 2 in Fig. 6 from Ref. [22]). Nevertheless, the high sensitivity of the proposed method makes it possible to easily register slightest changes in the conductance quantum states of DPCs even in the case of an inert component present in the analyzed medium. Interaction of an atom-sized PC with just one molecule of the dissolved gas can yield a resistivity change as large as at least $12.9 / n \mathrm{k} \Omega, n$ being the number of atoms in the contact. This value can easily be registered by most of the ordinary measuring devices.

A much richer picture can be seen when carbon dioxide is added to argon (Fig. 3b). In the case of pure argon, mostly the formation of samples with dimensions comparable to those of atom-sized contacts was observed, while carbon dioxide promotes the synthesis of more diverse structures of DPCs. The contacts formed under the action of the $\mathrm{Ar}-\mathrm{CO}_{2}$ mixture dissolved in water are characterized by conductivity channel diameters ranging from a few atoms to several hundreds of atoms. As a result, the quantum states of electric conductivity of DPCs synthesized in a medium containing carbon dioxide are drastically different from those presented by the conductance histogram for PCs grown in argon medium without carbon dioxide. The conductance histogram approach can thus allow one to efficiently identify the gases, even chemically inert ones.

To demonstrate the high sensitivity of the proposed detection principle, we significantly lowered the concentration of carbon dioxide in the mixture and found that this greatly affected the cyclic switchover process and the conductance in the metastable quantum states of DPCs (Fig. 3c).

A considerable change in the conductance histogram was observed for DPCs in an electrolyte exposed to gaseous methane. Methane is a hardly detectable substance, and the clearly observable response to the action of its low concentrations (Fig. 3d) is another confirmation of the high sensitivity of the proposed approach. Figure 3 clearly demonstrates that the histograms obtained for DPCs synthesized in four different gaseous media have pronounced qualitative and quantitative differences. In the simplest case, it is enough to know the position and intensity of the main maximum in the histogram to be able to identify the probed object. For a deeper analysis, parameters of the other extrema, like their amplitudes and widths, can be taken into consideration as well as the width of the whole histogram, its area, etc. By using a selection of parameters, it is possible to find even minor differences among the histograms. This approach allows creating data libraries which could be used to reliably detect single gases and gas mixtures and thereby implies absolute selectivity of the proposed method for the detection of gases.

This selectivity can easily be understood if we consider the quantum nature of the shell effect which clearly manifests itself in the conductance histograms. This effect determines the formation of metastable configurations of DPC characterized by quantized energy values of conductivity. The DPC nanostructure can thus be seen as a physical quantum system with a set of energy eigenstates corresponding to its local energy minima. It was shown that these states can group together into bunches of degenerate or close-lying levels called shells [18, 22, 27]. There is a clear relationship between the geometry of the DPC system shaped by the shell effect and its electrical properties: cross-sectional diameters of the DPC metallic channels correlate directly with their electrical transport parameters $[18,22,28,29]$. Conductance variations in a DPC structure account for the states of enhanced stability corresponding to certain PC diameters [22]. The plateaus in the stepped dependence $G(t)$ correspond to the metastable states of the contact. Each stable state changes to another through resistance (conductance) jumps, which thus produce stepped regions in the curve indicating a quantization effect (Fig. 2b). The minima and the maxima of the conductance of growing DPCs are reproduced in many cycles of a self-oscillatory process (defined before as the cyclic switchover effect). As a result, the proposed nanostructure can become an efficient tool to investigate quantum effects and their applications [22].

Our study demonstrates that the metastable states and related energy levels of DPC systems depend on the specific conditions under which they were formed. Every time 
we change the gaseous medium, we change the conditions of the experiment and thus create a new quantum DPC system with its own metastable states and energy levels. The dynamic regime of the experiments provided by the cyclic switchover synthesis of DPCs makes it possible to register the energy profile of the quantum system in the form of a conductance histogram. The energy profile of a DPC quantum system is its unique fingerprint that can be used to unambiguously identify the gaseous medium in which the DPCs are synthesized. The new concept of selective detection proposed here is based on the characterization of the interaction energy of a DPC structure with gaseous and liquid media through its quantum conductivity parameters. The quantum approach provides a wide choice of options for the development of a broad spectrum of novel methods and devices because the knowledge of energy parameters of any physicochemical system is a key factor for determination and prognosis of its properties.

Since quantum parameters are sensitive to nanoscale variations in the point contact configuration, the point contact quantum system can be used to precisely and reliably register those variations, even if they are caused by small concentrations of substances present in the probed gaseous medium, including chemically inert components like argon. The high sensitivity combined with a good reproducibility determines the high selectivity of the method, which also demonstrates some other advantages: the possibility of performing real-time measurements, simplicity and portability of devices which could be based on the method, and the large variety of gaseous and liquid substances which could be detected in this way. All these peculiarities establish solid ground for the development of new simple and inexpensive sensing technologies and devices.

Acknowledgements This work was partly supported by the NATO SPS Programme (Ref: SPS.MYP 985481) and the NAS of Ukraine. The authors thank Drs. O. G. Danylchenko and Christopher Ewels for helpful discussions and expert comments and V. A. Gudimenko and A. M. Pletnev for assistance in processing the results.

\section{Compliance with ethical standards}

Conflict of interest The authors declare that they have no conflict of interest.

\section{References}

1. Yang S, Jiang C, Wei SH (2017) Gas sensing in 2D materials. Appl Phys Rev 4:021304

2. Kaisti $M$ (2017) Detection principles of biological and chemical FET sensors. Biosens Bioelectron 98:437-448
3. Lim T, Lee J, Lee J, Ju S (2017) Detection of chemicals in water using a three-dimensional graphene porous structure as liquidvapor separation filter. Nano Res 10:971-979

4. Quesada-González D, Merkoçi A (2017) Mobile phone-based biosensing: an emerging "diagnostic and communication" technology. Biosens Bioelectron 92:549-562

5. Devadhasan JP, Kim D, Lee DY, Kim S (2017) Smartphone coupled handheld array reader for real-time toxic gas detection. Anal Chim Acta 984:168-176

6. Ali S, Khusro S (2016) Mobile phone sensing: a new application paradigm. Indian J Sci Technol 9:1-42

7. Piyare R (2013) Internet of things: ubiquitous home control and monitoring system using android based smart phone. Int J Internet Things 2:5-11

8. Patel UM, Padole KS (2018) Overview of MEMS sensors in automotive industry. Int J Eng Res Technol 7:160-163

9. Yang Y, Yang X, Tan Y, Yuan Q (2017) Recent progress in flexible and wearable bio-electronics based on nanomaterials. Nano Res 10:1560-1583

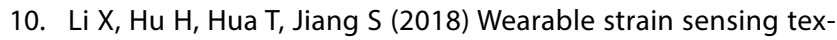
tile based on one-dimensional stretchable and weavable yarn sensors. Nano Res 99:99. https://doi.org/10.1007/s1227 4-018-2043-7

11. Fraden J (2016) Handbook of modern sensors: physics, designs, and applications, 5th edn. Springer, Heidelberg

12. Kamarchuk GV, Pospelov AP, Kamarchuk LV, Kushch IG (2015) Point-contact sensors and their medical applications for breath analysis: a review. In: Karachevtsev VA (ed) Nanobiophysics: fundamentals and applications. Pan Stanford Publishing, Singapore, pp 327-379

13. Priye A, Bird SW, Light YK, Ball CS, Negrete OA, Meaghe RJ (2017) A smartphone-based diagnostic platform for rapid detection of Zika, chikungunya, and dengue viruses. Sci Rep 7:44778

14. Gumpu MB, Sethuraman S, Krishnan UM, Rayappan JBB (2015) $A$ review on detection of heavy metal ions in water-an electrochemical approach. Sens Actuators B 213:515-533

15. Varghese SS, Lonkar S, Singh KK, Swaminathan S, Abdala A (2015) Recent advances in graphene based gas sensors. Sens Actuators B 218:160-183

16. Naidyuk YG, Yanson IK (2005) Point-contact spectroscopy. Springer, New York

17. Krans JM, van Ruitenbeek JM, Fisun VV, Yanson IK, de Jongh LJ (1995) The signature of conductance quantization in metallic point contacts. Nature 375:767-769

18. Yanson Al, Yanson IK, van Ruitenbeek JM (1999) Observation of shell structure in sodium nanowires. Nature 400:144-146

19. Mares Al, van Ruitenbeek JM (2005) Observation of shell effects in nanowires for the noble metals $\mathrm{Cu}, \mathrm{Ag}$, and $\mathrm{Au}$. Phys Rev B 72:205402

20. Pospelov AP, Kamarchuk GV, Alexandrov YL, Zaika AS, Yeremenko AV, Faulques E (2004) New development of impedance spectroscopy. In: Faulques EC, Perry DL, Yeremenko AV (eds) Spectroscopy of emerging materials (NATO Science Series II). Kluwer, Dordrecht, pp 331-338

21. Kamarchuk GV, Pospelov AP, Savitsky AV, Koval' LV (2014) Nonlinear cyclic transport phenomena in copper point contacts. Low Temp Phys 40:1198-1205

22. Pospelov AP, Pilipenko Al, Kamarchuk GV, Fisun VV, Yanson IK, Faulques $E$ (2015) A new method for controlling the quantized growth of dendritic nanoscale point contacts via switchover and shell effects. J Phys Chem C 119:632-639

23. Pilipenko AI, Pospelov AP, Kamarchuk GV, Bondarenko IS, Shablo AA, Bondarenko SI (2011) Point-contact sensory nanostructure modeling. Funct Mater 18:324-327

24. Pospelov AP, Kamarchuk GV, Savytskyi AV, Sakhnenko MD, Ved MV, Vakula VL (2017) Macroscopic simulation of atom-sized 
structures of functional materials: phenomenology of the elongated electrode system. Funct Mater 24:463-468

25. Kulik IO, Omelyanchuk AN, Shekhter RI (1977) Electrical conductivity of point microcontacts and the spectroscopy of phonons and impurities in normal metals. Sov J Low Temp Phys 3:1543-1558

26. Kamarchuk GV, Pospelov OP, Yeremenko AV, Faulques E, Yanson IK (2006) Point-contact sensors: new prospects for a nanoscale sensitive technique. Europhys Lett 76:575-581

27. Martin TP (1996) Shells of atoms. Phys Rep 273:199-241

28. Glazman LI, Lesovik GB, Khmel'nitskii DE, Shekhter RI (1988) Reflectionless quantum transport and fundamental ballistic-resistance steps in microscopic constrictions. JETP Lett 48:238-241

29. Obermair C, Kuhn H, Schimmel Th (2011) Lifetime analysis of individual-atom contacts and crossover to geometric-shell structures in unstrained silver nanowires. Beilstein J Nanotechnol 2:740-745

Publisher's Note Springer Nature remains neutral with regard to jurisdictional claims in published maps and institutional affiliations. 